\title{
APARÊNCIA ECOLÓGICA E CONSERVAÇÃO DE ESPÉCIES LENHOSAS PELOS MAKUXIS NA SAVANA DE RORAIMA, AMAZÔNIA BRASILEIRA
}

\section{ECOLOGICAL APPEARANCE AND WOODY PLANTS CONSERVATION BY MAKUXI INDIGENOUS PEOPLE IN THE RORAIMA SAVANNA, BRAZILIAN AMAZON}

\author{
Rodrigo Leonardo Costa de Oliveira ${ }^{1 *}$, Luís Felipe Paes de Almeida²; Maria Fernanda Berlingieri Durigan ${ }^{3}$, \\ Veridiana Vizoni Scudeller ${ }^{4}$, Reinaldo Imbrozio Barbosa ${ }^{5}$ \\ ${ }^{1 *}$ Coordenação de Ciências Biológicas, Universidade Estadual de Roraima; ${ }^{2}$ Instituto Insikiran, Universidade Federal de Roraima; ${ }^{3}$ Embrapa Roraima; ${ }^{4}$ Instituto \\ de Ciências Biológicas, Universidade Federal do Amazonas; ${ }^{5}$ Instituto Nacional de Pesquisas da Amazônia, Núcleo de Pesquisas de Roraima. \\ *rodrigo@uerr.edu.br
}

\section{RESUMO}

A hipótese da aparência ecológica em estudos etnobotânicos está baseada na relação oferta/procura entre populações humanas e os recursos de seu meio, com a premissa de que as plantas com maior disponibilidade no ambiente (aparentes) seriam as mais reconhecidas por sua utilidade dentro de uma determinada cultura. $\mathrm{O}$ presente estudo avaliou esta hipótese em relação às espécies lenhosas nativas registradas nos ambientes florestal (Buritizal, Mata Ciliar e Ilha de Mata) e não florestal (Lavrado) na Comunidade Indígena Darora, etnia Makuxi, na Terra Indígena São Marcos, Roraima. Foram realizadas entrevistas semiestruturadas com 60 participantes (36 homens e 24 mulheres), de 38 famílias, entre 18 e 84 anos de idade. Também foi realizado um levantamento fitossociológico para verificar a disponibilidade de recursos lenhosos em oito parcelas ( 0,25 ha cada), instaladas a diferentes distâncias do centro da comunidade. As espécies com os maiores valores de uso (VU) da comunidade em geral foram Mauritia flexuosa, Copaifera pubiflora, Byrsonima crassifolia e B. coccolobifolia. A hipótese da aparência ecológica foi corroborada nos ambientes Lavrado e Buritizal. Houve correlação positiva entre os parâmetros fitossociológicos e o valor de uso nas categorias Alimentação e Combustível no ambiente Lavrado, nas categorias Alimentação, Artesanato e Construção no ambiente Buritizal, e nas categorias Medicinal e Tecnologia no ambiente Ilha de Mata.

Palavras-chave: Valor de Uso; Buritizal; Mata Ciliar; Ilha de Mata; Lavrado.

\begin{abstract}
The ecological appearance hypothesis in ethnobotanical studies is based on the supply/demand relationship between human populations and environmental resources, with the premise that those plants with higher environmental availability (apparent) would be most recognized for their utility within a particular culture. This study evaluated the ecological appearance hypothesis of native woody plants recorded in the forest and non-forest vegetation types in the Darora Indigenous Community, ethnic group Makuxi, in the São Marcos Indigenous Land, state of Roraima. Semi-structured interviews were carried out with 60 participants ( 36 men and 24 women) from 38 families between 18 and 84 years old. A phytosociological survey was made to evaluate woody resources availability in eight plots installed with 0.25 ha each, with different distances from the community center. The species with highest UV were Mauritia flexuosa, Copaifera pubiflora, Byrsonima crassifolia and B. coccolobifolia. Ecological appearance was corroborated in Lavrado and Buritizal vegetation types. Food and Fuel categories were found in Lavrado; Food, Crafts and Construction categories were found in Buritizal, whereas Medicinal and Technology categories were found in the Forest Island.
\end{abstract}

Keywords: Use Value; Mauritia palm swamp; Alluvial forest; Forest Island; Lavrado.

\section{INTRODUÇÃO}

$\mathrm{Na}$ perspectiva ecológica dos estudos etnobotânicos, a hipótese da aparência ecológica está baseada na relação oferta/procura entre populações humanas e os recursos de seu meio, utilizando-se técnicas etnobotânicas associadas a dados ecológicos (LUCENA et al., 2012). O estudo investiga, portanto, a premissa de que as plantas com maior visibilidade em um determinado ambiente seriam as 
mais percebidas, mediante características ecológicas como abundância, distribuição e área basal e, consequentemente, reconhecidas por sua utilidade dentro de uma determinada cultura (PHILLIPS e GENTRY, 1993a, 1993b; ALBUQUERQUE e LUCENA, 2005).

A hipótese da aparência ecológica foi testada primeiramente por Phillips e Gentry (1993a, 1993b) em Tambopata, na Amazônia peruana, na qual os autores elaboraram uma medida quantitativa (valor de uso - VU) que representava a importância relativa das espécies de acordo com o número de citações de uso dos entrevistados. Em complemento, os resultados obtidos por meio dos parâmetros fitossociológicos (densidade, dominância e frequência) da vegetação local indicaram as espécies mais disponíveis no ambiente. Os dados do VU e dos parâmetros fitossociológicos foram confrontados e, desta forma, esperava-se que as plantas mais citadas fossem as mais disponíveis, corroborando a hipótese.

A aplicação desta técnica permitiu nos últimos anos reconhecer diferenças entre o conhecimento local de homens e mulheres, espécies potencialmente em perigo de extinção local e áreas altamente sobre-exploradas, servindo como base para planos de manejo e conservação ambiental (FERRAZ et al., 2006; LUCENA et al., 2007; ALBUQUERQUE, 2010). Embora tenha apresentado limitações quanto ao emprego do VU e à forma de inventariar a vegetação local, a hipótese da aparência ecológica foi confirmada em diferentes ambientes, como a Floresta Amazônica (GALEANO, 2000), áreas de savana na África (AYANTUNDE et al., 2009), florestas secas (MALDONADO et al., 2013) e manguezais (JIMÉNEZ-ESCOBAR e RANGEL, 2012), embora tenha sido refutada em outras localidades, como na Caatinga do nordeste brasileiro (LUCENA et al., 2012), nos Andes bolivianos (BRANDT et al., 2012) e em florestas tropicais (TORRE-CUADROS e ISLEBE, 2003; CUNHA e ALBUQUERQUE, 2006). Embora não tenha sido corroborada em todos os ambientes que foi testada, percebemos que a aplicação da hipótese da aparência ecológica implicou em direcionamentos quanto à compreensão da utilização e conservação das espécies vegetais pelas respectivas culturas.

Um caso relevante para se testar esta relação entre a aparência ecológica e utilização dos recursos remete às áreas de savana do extremo norte da Amazônia brasileira, onde se encontram inúmeras populações indígenas que se relacionam tradicionalmente com o recurso vegetal disponível. Esta relação entre ambiente e populações humanas nesta região da Amazônia é pobremente estudada, apesar dos recursos naturais estarem sofrendo grande erosão devido ao avanço contínuo e em larga escala de lavouras agrícolas, como soja e arroz irrigado (CORDEIRO, 2005; GIANLUPPI e SMIRDELE, 2005) e a introdução de espécies florestais exóticas (AGUIAR et al., 2014). Essa região abriga a Terra Indígena Raposa/Serra do Sol e a Terra Indígena São Marcos, caracterizadas por suas grandes extensões territoriais, nas quais são encontradas as etnias Makuxi, Wapixana, Ingarikó, Taurepáng, Patamona e Sapará (PINHO et al., 2010). A região apresenta um complexo de diferentes fitofisionomias, que vão desde ambientes não florestais (savanas típicas de vegetação aberta) a ambientes florestais (Mata Ciliar, Buritizal e Ilhas de Mata) (BARBOSA et al., 2007).

O presente estudo avaliou a hipótese da aparência ecológica de espécies lenhosas nativas registradas nos ambientes florestal (Buritizal, Mata Ciliar e Ilha de Mata) e não florestal (Lavrado), associando a importância ecológica das espécies lenhosas e sua utilidade na comunidade Darora situada em uma área de savana da Terra Indígena São Marcos (TISM), inferindo sobre o conhecimento botânico local.

\section{MATERIAL E MÉTODOS \\ Área de estudo}

A Terra Indígena São Marcos (TISM) compreende 654.110 ha e possui 42 comunidades indígenas das etnias Makuxi, Taurepáng e Wapixana. O estudo envolveu um grupo da etnia Makuxi, da Comunidade Darora, que vive em uma região conhecida como Baixo São Marcos (310'42 "N; 60²3'34" W). A área de estudo localiza-se a $90 \mathrm{~km}$ da sede da capital, Boa Vista, Estado de Roraima (Figura 1). O clima local é tropical de savana (Aw), de acordo com a classificação de Köppen, com temperatura média anual de $27,8^{\circ} \mathrm{C}$ e precipitação média anual de $\sim 1.650 \mathrm{~mm}$. O período mais seco ocorre entre os meses de dezembro e março ( $\pm 9 \%$ precipitação anual), e os meses mais chuvosos são entre maio e agosto ( $\pm 70 \%$ precipitação anual) (BARBOSA, 1997).

A ocupação do local onde está a Comunidade Darora ocorreu por volta de 1941, quando algumas famílias da Comunidade Xumina (Terra Indígena Raposa/Serra do Sol) migraram para a região por causa da dificuldade em encontrar áreas para a agricultura. A comunidade tem uma forte aptidão para a agricultura e pecuária, entendida por eles como necessária para o alimento e sustento. $\mathrm{O}$ extrativismo 
ainda é usado e está sempre relacionado com as necessidades para a construção de casas, cercas e coleta de frutos para o alimento.

Existem duas escolas públicas na Comunidade Darora: uma para o ensino infantil, mantidos pela Prefeitura de Boa Vista, e uma para o ensino fundamental e médio, mantida pelo Governo do Estado. Todos os moradores falam fluentemente o português, mas a língua makuxi, tronco linguístico Karib, é ensinado na pré-escola. No entanto, a língua nativa é raramente falada pelos moradores, com apenas dois professores e um idoso falantes.

\section{Ética e aspectos legais}

Este estudo faz parte do projeto de pesquisa "Uso e conservação dos recursos vegetais por comunidades indígenas no norte de Roraima, Amazônia Setentrional", do Programa de Pós-graduação em Ciências Biológicas (Botânica), do Instituto Nacional de Pesquisas da Amazônia (PPGBot-INPA) e Universidade Estadual de Roraima (UERR). Ele foi submetido e aprovado pelo Comitê de Ética em Pesquisa com Seres Humanos do Instituto Nacional de Pesquisas da Amazônia/Comissão Nacional de Ética em Pesquisa com Seres Humanos (CEP-INPA/CONEP), com o número de autorização 814.370 2014. O estudo foi autorizado pela Fundação Nacional do Índio (FUNAI), número do processo 08.620.002869/2014-15, e pelo Instituto Nacional de Patrimônio Histórico e Artístico Natural (IPHAN), número do processo 01.450.001678/2014-88.

\section{Levantamento etnobotânico}

Para a coleta de dados etnobotânicos, foram realizadas entrevistas semiestruturadas com 60 participantes ( 36 homens e 24 mulheres) de 38 famílias, entre 18 e 84 anos de idade. Todas as famílias da comunidade foram visitadas e cada morador foi convidado a participar da pesquisa. O objetivo da pesquisa foi explicado e àqueles que concordaram em participar da entrevista lhes foi dada uma cópia do Termo de Consentimento Livre e Esclarecido (TCLE). As entrevistas ocorreram entre novembro de 2014 a novembro de 2015. Em primeiro lugar, os entrevistados indicaram locais de coleta das espécies utilizadas em áreas não florestais e florestais da savana, e, em seguida, foi utilizada a técnica da listalivre, na qual eles citavam as espécies que conheciam, como sugerido por Albuquerque et al. (2014). Em um segundo momento, foram realizadas entrevistas semiestruturadas (ALBUQUERQUE et al., 2014) com perguntas sobre coleta, formas de uso e partes de plantas utilizadas. As respostas foram agrupadas nas categorias Alimentação, Ração Animal. Artesanato, Combustível, Construção, Medicinal e Tecnologia adaptadas a partir de estudos anteriores (LINS NETO et al., 2008; LUCENA et al., 2012).

\section{Levantamento ecológico}

Utilizou-se um levantamento florístico associado com técnicas fitossociológicas para verificar a disponibilidade de recursos lenhosos para a Comunidade Indígena Darora. Desta forma, oito parcelas ( 0,25 ha cada) foram instaladas em diferentes distâncias do centro da comunidade: quatro na área típica de savana (não florestal) e que neste estudo chamamos Lavrado, e outras quatro em três áreas florestais: (i) duas na Mata Ciliar do rio Takutu, (ii) uma em um Buritizal, localmente designado no igarapé Maracajá, e (iii) uma em Ilha de Mata (Figura 1).

A localização de cada parcela foi previamente estabelecida durante as reuniões com os residentes, de acordo com o uso e disponibilidade dos recursos florestais comunitários. Cada parcela foi dividida em 10 subparcelas de $25 \mathrm{~m}$ x $10 \mathrm{~m}$ cada. Nas parcelas do Lavrado (não florestal), toda árvore com diâmetro de base $\geq 2 \mathrm{~cm}$ foi medida a $2 \mathrm{~cm}$ de altura do solo $\left(\mathrm{DSH}_{2} \mathrm{~cm} \geq 2 \mathrm{~cm}\right)$, seguindo metodologia especificada por Barbosa et al. (2005). Nas parcelas florestais, foram medidos todos os indivíduos com DAP $\geq 10 \mathrm{~cm}$. Para cada tipo de vegetação (não florestal e florestal), parâmetros fitossociológicos foram calculados (Densidade Relativa - DR, Dominância Relativa - DoR, Frequência Relativa - FR e Valor de Importância - VI). 

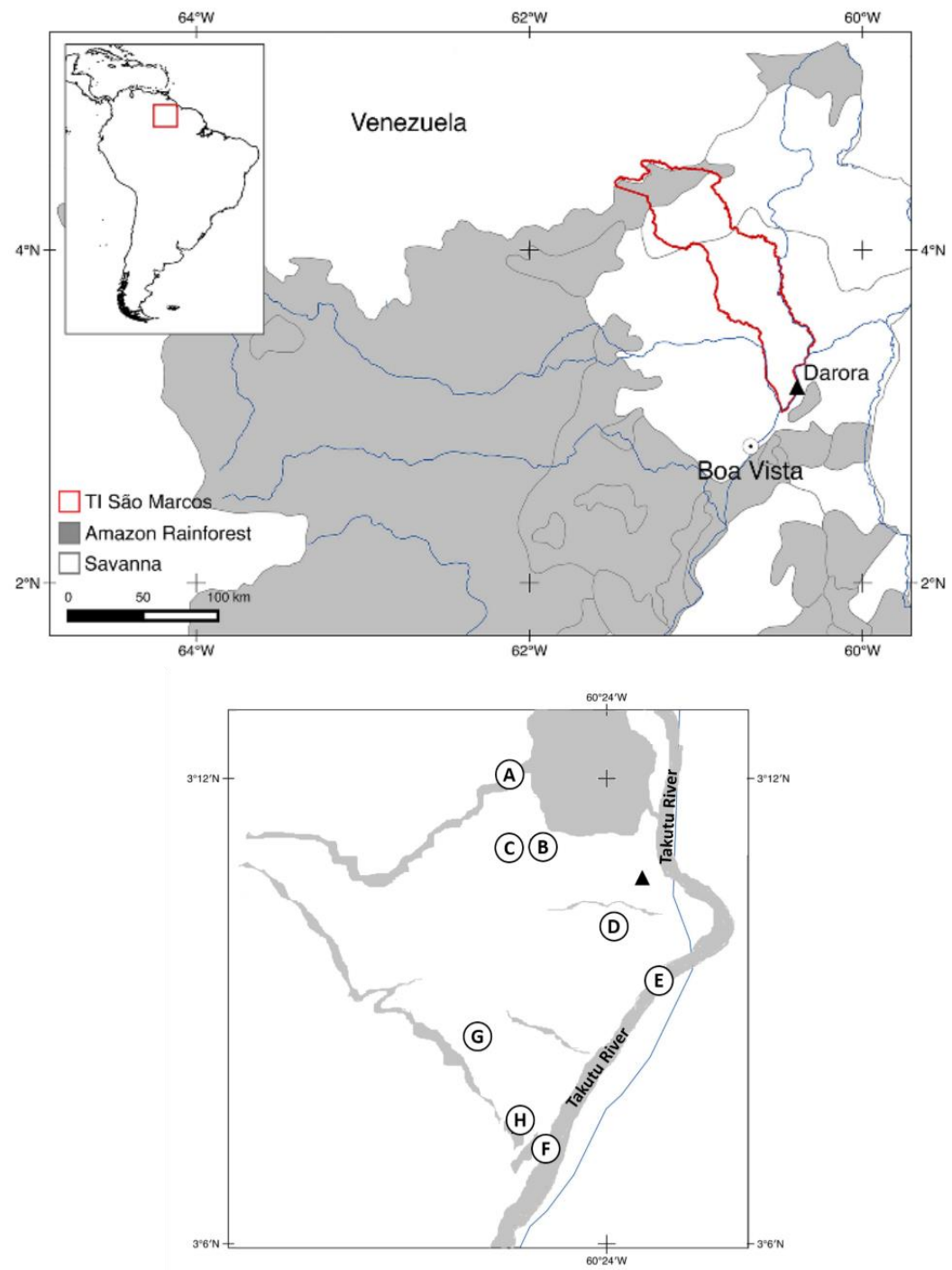

Figura 1: Localização da Comunidade Darora - Terra Indígena São Marcos (TISM), Roraima, Brasil. Localização das parcelas no ambiente não florestal (Lavrado - C, B, D e G); no ambiente florestal (Buritizal - A, Mata Ciliar - E e F, Ilha de Mata - H). Fonte: Oliveira (2016).

\section{Coleta do material botânico}

Amostras das espécies foram coletadas e a identificação taxonômica foi realizada por meio de comparações mediante auxílio de parabotânicos, especialistas e bibliografias específicas (RIBEIRO et al, 1999; MELO e BARBOSA, 2007; FLORES e RODRIGUES, 2010; WITTMANN et al., 2010). As amostras foram incorporadas ao acervo do Herbário UFRR da Universidade Federal de Roraima, Boa Vista, Roraima. A classificação utilizada seguiu o estabelecido no sistema APG IV (2016).

\section{Análise dos dados}

Em cada ambiente, foram calculados o valor de uso total $\left(\mathrm{VU}=\sum \mathrm{Ui} / \mathrm{n}\right)$ de cada espécie, e o valor de uso de cada espécie em cada categoria de uso em que esta determinada espécie foi indicada $\left(\mathrm{VUc}=\sum \mathrm{VUc} / \mathrm{nc}\right)$. A técnica do valor de uso $(\mathrm{VU})$ foi primeiramente proposta por Phillips e Gentry (1993a, 1993b) e adaptada por Rossato et al. (1999), onde: Ui = número de usos mencionados por cada informante para a espécie; $\mathrm{n}=$ número total de informantes; $\mathrm{VUc}=$ número de usos mencionado por cada participante para a espécie em uma determinada categoria de uso; nc = número de espécies numa determinada categoria de uso. O valor de uso (VU) permite avaliar as espécies mais importantes, indicando quais espécies são mais mencionadas pelos informantes. Por outro lado, tais medidas expressam apenas o que as pessoas dizem conhecer e não o que efetivamente é usado naquele momento (ALBUQUERQUE e LUCENA, 2005; REYES-GARCÍA et al., 2006).

Para testar a hipótese da aparência ecológica foram utilizadas as espécies amostradas em ambos os levantamentos (etnobotânico e fitossociológico), utilizando-se a correlação de Pearson, bem como 
análises de regressão linear simples entre os parâmetros fitossociológicos de cada espécie (densidade relativa, dominância relativa, frequência relativa e valor de importância) de acordo com o tipo de ambiente da savana (não florestal ou florestal) e os índices etnobotânicos das espécies (valor de uso e valor de uso da categoria). Por apresentar número de espécies e respectivos valores reduzidos, a categoria Ração Animal foi incluída na categoria Alimentação para a análise dos dados.

\section{RESULTADOS}

Das 52 espécies citadas no levantamento fitossociológico, apenas 36 foram consideradas úteis. Destas, 9 espécies foram registradas nos ambientes não florestais (Lavrado) e 31 espécies foram registradas nos ambientes florestais, sendo 10 espécies no Buritizal, 19 na Mata Ciliar e 8 na Ilha de Mata (Tabelas 1-4). Quatro espécies típicas do ambiente Lavrado foram registradas entre os ambientes florestais, Curatella americana, registrada em todos os ambientes florestais e Bowdichia virgilioides, Byrsonima crassifolia e Himatanthus drasticus registradas na Ilha de Mata. Quatro vernáculos foram citados para duas espécies cada. O vernáculo mirixi foi designado para as espécies Byrsonima crassifolia e B. coccolobifolia, assim como a goiabinha (Myrcia sp. e Eugenia sp.), envireira (Guatteria sp. e Xylopia aromatica) e ucuuba (Virola molissima e $V$. surinamensis). Neste caso, os cálculos do valor de uso foram tabulados para as seis espécies em questão.

Todas as espécies apresentaram o Valor de Importância (VI) > 1 nos quatro ambientes, ou seja, das espécies citadas pela comunidade todas se apresentaram ecologicamente importantes. As espécies com os maiores valores de VI em todos os ambientes foram: Byrsonima crassifolia, B. coccolobifolia, Mauritia flexuosa e Etabalia sp. (Tabelas 1-4). Destas, apenas Etabalia sp. não apresentou um alto valor de uso (VU). As espécies com os maiores VU da comunidade em geral foram o buriti (Mauritia flexuosa, $\mathrm{VU}=3,37$ ), a espécie mais importante; seguido pela copaíba (Copaifera pubiflora, $\mathrm{VU}=3,23$ ) e pelos mirixis (Byrsonima crassifolia e B. coccolobifolia, $\mathrm{VU}=2,50$ ). Copaifera pubiflora apresentou $\mathrm{VI}=7,94$ na Mata Ciliar e VI = 26,71 na Ilha de Mata, apresentando maior destaque no segundo tipo de ambiente. No Buritizal, Xylopia aromatica apresentou apenas um indivíduo, assim como Bixa arborea, Couepia multiflora, Hydrochorea corymbosa e Myrcia sp. no ambiente Mata Ciliar, e ecologicamente consideradas "espécies raras".

Tabela 1: Espécies registradas no ambiente Lavrado em ordem decrescente do VU. N - número de indivíduos; DR - Densidade relativa; DoR - Dominância Relativa; FR - Frequência relativa, VI - Valor de Importância; VU - Valor de uso; UFRR - número de registro no Herbário UFRR.

\begin{tabular}{lllllllll}
\hline Lavrado Espécies & Nome comum & N & DR & DoR & FR & VI & VU & UFRR \\
\hline Byrsonima coccolobifolia Kunth. & mirixi & 163 & 22.99 & 23.14 & 22.73 & 68.86 & 2.5 & 8483 \\
Byrsonima crassifolia (L.) Kunth. & mirixi & 268 & 37.80 & 51.06 & 24.68 & 113.54 & 2.5 & 8482 \\
Curatella americana L. & caimbé & 18 & 2.54 & 8.97 & 8.44 & 19.95 & 1.45 & 8446 \\
Bowdichia virgilioides Kunth. & paricarana & 19 & 2.68 & 4.00 & 9.09 & 15.77 & 1.38 & 8471 \\
Himatanthus drasticus (Mart.) Plumel & sucuba & 17 & 2.40 & 2.07 & 5.84 & 10.31 & 0.91 & 8487 \\
Palicourea rigida Kunth. & douradão & 17 & 2.40 & 0.60 & 7.79 & 10.79 & 0.63 & 8489 \\
Roupala montana Aubl. & congonha & 81 & 11.42 & 4.78 & 6.49 & 22.69 & 0.61 & 8484 \\
Byrsonima verbascifolia (L.) DC. & orelha-de-burro & 19 & 2.68 & 0.78 & 8.44 & 11.9 & 0.31 & 8480 \\
Antonia ovata Pohl. & timbó & 107 & 15.09 & 4.60 & 6.49 & 26.18 & 0.13 & 8485 \\
\hline
\end{tabular}

Entre as categorias de uso (Tabela 5), a categoria Construção apresentou um grande número de espécies no Buritizal (52\%) e na Mata Ciliar (72\%), porém no ambiente Lavrado apenas o caimbé (Curatella americana) recebeu citações de uso. A categoria Alimentação foi citada nos quatro ambientes (44\%), destacando as espécies de mirixi, buriti, jenipapo (Genipa americana) e taperebá (Spondias mombin). A categoria Combustível apresentou destaque com as espécies de mirixis no ambiente Lavrado e Ilha de Mata (56\%). A categoria Medicinal teve maior destaque nos ambientes Lavrado e Ilha de Mata (68\%), com o caimbé, a copaíba, a sucuba (Himatanthus drasticus) e as espécies de mirixi. A categoria Tecnologia teve maior destaque pelo número de espécies num ambiente, Illha de Mata (63\%), com a copaíba e paricarana (Bowdichia virgilioides), mas o maior VU na categoria foi da manga-braba (Andira sp.) e do caimbé. A categoria Artesanato apresentou apenas sete espécies (19\% do total), e no ambiente Lavrado não obteve nenhuma citação (Tabela 5).

A copaíba (Copaifera pubiflora) e o mari-mari (Cassia moschata) foram as únicas espécies associadas a todas as categorias de uso, embora ambas tenham se destacado principalmente na categoria 
Construção. A copaíba também teve grande destaque na categoria Medicinal e o mari-mari na categoria Tecnologia.

Tabela 2: Espécies registradas no ambiente Buritizal em ordem decrescente do VU. $\mathrm{N}$ - número de indivíduos; DR - Densidade relativa; DoR - Dominância Relativa; FR - Frequência relativa, VI - Valor de Importância; VU - Valor de uso; UFRR - número de registro no Herbário UFRR.

\begin{tabular}{lllllllll}
\hline Buritizal Espécies & Nome comum & N & DR & DoR & FR & VI & VU & UFRR \\
\hline Mauritia flexuosa L. f. & buriti & 27 & 18 & 37.8 & 7.14 & 62.94 & 3.37 & - \\
Curatella americana L. & caimbé & 1 & 0.67 & 0.39 & 1.42 & 2.48 & 1.45 & 8446 \\
Euterpe precatoria Mart. & açaí & 17 & 11.3 & 3.76 & 7.14 & 22.2 & 0.72 & - \\
Virola molissima Poepp. ex. A. DC. & ucuuba & 11 & 7.33 & 2.88 & 5.71 & 15.92 & 0.70 & 8430 \\
Virola surinamensis & ucuuba & 5 & 3.33 & 2.83 & 5.71 & 11.87 & 0.70 & 8635 \\
(Rol. ex Rottb.) Warb. & mirixi-de-galega & 3 & 2 & 1.92 & 1.42 & 5.34 & 0.50 & 8443 \\
Byrsonima sp. & miri & 6 & 4 & 3.64 & 7.14 & 14.78 & 0.43 & 8429 \\
Humiria balsamifera Aubl. & tento & 14 & 9.33 & 5.88 & 10 & 25.21 & 0.12 & 8437 \\
Ormosia smithii Rudd. & envireira & 10 & 6.67 & 3.82 & 10 & 20.49 & 0.10 & 8442 \\
Guatteria sp. & envireira & 1 & 0.67 & 0.26 & 1.42 & 2.35 & 0.10 & 8449 \\
Xylopia aromatica (Lam.) Mart. & louro & 14 & 9.33 & 6.75 & 10 & 26.08 & 0.02 & 8432 \\
Endlicheria sp. & & & & & & & & \\
\hline
\end{tabular}

Tabela 3: Espécies registradas no ambiente Mata Ciliar em ordem decrescente do VU. N - número de indivíduos; DR - Densidade relativa; DoR - Dominância Relativa; FR - Frequência relativa, VI - Valor de Importância; VU - Valor de uso; UFRR - número de registro no Herbário UFRR.

\begin{tabular}{lllllllll}
\hline Mata Ciliar Espécies & Nome comum & N & DR & DoR & FR & VI & VU & UFRR \\
\hline Copaifera pubiflora Benth. & copaíba & 2 & 1.98 & 4.33 & 1.63 & 7.94 & 3.23 & 8454 \\
Genipa americana L. & jenipapo & 10 & 9.9 & 3.21 & 14.75 & 27.86 & 1.67 & 8488 \\
Curatella americana L. & caimbé & 7 & 6.93 & 3.69 & 4.91 & 15.53 & 1.45 & 8446 \\
Spondias mombin & taperebá & 3 & 2.97 & 3.2 & 3.27 & 9.44 & 0.97 & - \\
Eschweilera sp. & macaco-aricuia & 3 & 2.97 & 1.26 & 3.27 & 7.5 & 0.95 & 8463 \\
Andira sp. & manga-braba & 2 & 1.98 & 2.75 & 3.27 & 8 & 0.77 & 8465 \\
Cassia moschata Kunth. & mari-mari & 3 & 2.97 & 3.02 & 3.27 & 9.26 & 0.68 & 8452 \\
Cassia sp. & jurema & 16 & 15.84 & 3.27 & 6.55 & 25.66 & 0.45 & 8456 \\
Eugenia sp. & goiabinha & 2 & 1.98 & 1.29 & 3.27 & 6.54 & 0.35 & 8464 \\
Myrcia sp. & goiabinha & 1 & 0.99 & 0.21 & 1.63 & 2.83 & 0.35 & 8453 \\
Hydrochorea corymbosa (Rich.) & vassourinha & 1 & 0.99 & 1.78 & 1.63 & 4.4 & 0.32 & 8468 \\
Barneby \& J.W.Grimes & maria-preta & 8 & 7.92 & 23.9 & 9.83 & 41.65 & 0.28 & 8470 \\
Vitex schomburgkiana Schauer & mututi & 20 & 19.8 & 31.07 & 13.11 & 63.98 & 0.12 & 8459 \\
Etabalia sp. & piritón & 3 & 2.97 & 1.05 & 3.27 & 7.29 & 0.12 & 8478 \\
Swartzia sp. & uixi & 1 & 0.99 & 1.67 & 1.63 & 4.29 & 0.08 & 8450 \\
Couepia multiflora Benth. & samaúma & 2 & 1.98 & 0.52 & 3.27 & 5.77 & 0.07 & - \\
Ceiba sp. & copaibinha & 8 & 7.92 & 8.35 & 4.91 & 21.18 & 0.05 & 8460 \\
Cynometra bauhinifolia Benth. & urucum-brabo & 1 & 0.99 & 0.21 & 1.63 & 2.83 & 0.02 & 8467 \\
Bixa arborea Huber & & & & & & & & \\
\hline
\end{tabular}

Tabela 4: Espécies registradas no ambiente Ilha de Mata em ordem decrescente do VU. N - número de indivíduos; DR - Densidade relativa; DoR - Dominância Relativa; FR - Frequência relativa, VI - Valor de Importância; VU - Valor de uso; UFRR - número de registro no Herbário UFRR.

\begin{tabular}{lllllllll}
\hline Ilha de Mata Espécies & Nome comum & N & DR & DoR & FR & VI & VU & UFRR \\
\hline Copaifera pubiflora Benth. & copaíba & 4 & 6.06 & 14.77 & 5.88 & 26.71 & 3.23 & 8454 \\
Byrsonima crassifolia $($ L.) Kunth. & mirixi & 1 & 1.52 & 0.31 & 2.94 & 4.77 & 2.50 & 8482 \\
Genipa americana L. & jenipapo & 3 & 4.55 & 2.33 & 5.88 & 12.76 & 1.67 & 8488 \\
Curatella americana L. & caimbé & 18 & 27.27 & 25.78 & 17.64 & 70.69 & 1.45 & 8446 \\
Bowdichia virgilioides Kunth. & paricarana & 4 & 6.06 & 3.36 & 5.88 & 15.3 & 1.38 & 8471 \\
Himatanthus drasticus (Mart.) Plumel & sucuba & 2 & 3.03 & 2.66 & 5.88 & 11.57 & 0.92 & 8487 \\
Anadenanthera peregrina $($ L.) Speg. & angico & 4 & 6.06 & 3.45 & 8.82 & 18.33 & 0.78 & 8475 \\
Eugenia sp. & goiabinha & 6 & 9.09 & 7.25 & 8.82 & 25.16 & 0.35 & 8464 \\
\hline
\end{tabular}


Tabela 5: Valor uso das categorias de uso das espécies registradas nos ambientes Lavrado, Buritizal, Mata Ciliar e Ilha de Mata na Comunidade Darora, Boa Vista, Roraima.

\begin{tabular}{|c|c|c|c|c|c|c|c|c|}
\hline & Espécies & 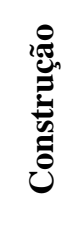 & 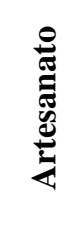 & 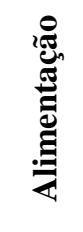 & 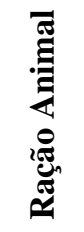 & 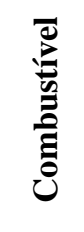 & 馬 & 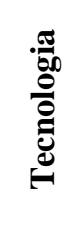 \\
\hline \multirow{9}{*}{ 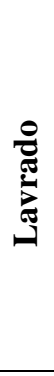 } & Antonia ovata & 0 & 0 & 0 & 0 & 0 & 0 & 0.24 \\
\hline & Bowdichia virgilioides & 1 & 0 & 0 & 0 & 0.33 & 0.44 & 0.15 \\
\hline & Byrsonima coccolobifolia & 0 & 0 & 1.46 & 0 & 1.67 & 1.18 & 0.15 \\
\hline & Byrsonima crassifolia & 0 & 0 & 1.46 & 0 & 1.67 & 1.18 & 0.15 \\
\hline & Byrsonima verbascifolia & 0 & 0 & 0.16 & 0 & 0 & 0.33 & 0 \\
\hline & Curatella americana & 0.06 & 0 & 0 & 0.05 & 0.07 & 1.67 & 0.50 \\
\hline & Himatanthus drasticus & 0 & 0 & 0 & 0 & 0.04 & 1.31 & 0.03 \\
\hline & Palicourea rigida & 0 & 0 & 0 & 0 & 0 & 0.97 & 0 \\
\hline & Roupala montana & 0 & 0 & 0.03 & 0 & 0.11 & 0.85 & 0 \\
\hline \multirow{19}{*}{ 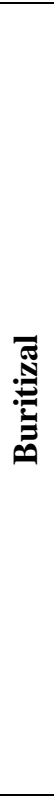 } & Alchornea sp. & 0 & 0 & 0 & 0 & 0 & 0 & 0 \\
\hline & Amanoa guianensis & 0 & 0 & 0 & 0 & 0 & 0 & 0 \\
\hline & Buchenavia capitata & 0 & 0 & 0 & 0 & 0 & 0 & 0 \\
\hline & Byrsonima sp. & 0.18 & 0 & 0.35 & 0 & 0.26 & 0.03 & 0 \\
\hline & Caryocar microcarpum & 0 & 0 & 0 & 0 & 0 & 0 & 0 \\
\hline & Curatella americana & 0.06 & 0 & 0 & 0.05 & 0.07 & 1.67 & 0.50 \\
\hline & Endlicheria sp. & 0.02 & 0 & 0 & 0 & 0 & 0 & 0 \\
\hline & Euterpe precatoria & 0.37 & 0 & 0.59 & 0 & 0 & 0.05 & 0 \\
\hline & Guatteria sp. & 0.12 & 0 & 0 & 0 & 0 & 0 & 0 \\
\hline & Hirtella paniculata & 0 & 0 & 0 & 0 & 0 & 0 & 0 \\
\hline & Humiria balsamifera & 0.53 & 0 & 0 & 0 & 0 & 0 & 0 \\
\hline & Malouetia sp. & 0 & 0 & 0 & 0 & 0 & 0 & 0 \\
\hline & Mauritia flexuosa & 1.86 & 2.33 & 1.81 & 0 & 0.04 & 0 & 0.32 \\
\hline & Ormosia smithii & 0 & 0.50 & 0 & 0 & 0.04 & 0 & 0 \\
\hline & Tapirira guianensis & 0 & 0 & 0 & 0 & 0 & 0 & 0 \\
\hline & Trattinickia rhoifolia & 0 & 0 & 0 & 0 & 0 & 0 & 0 \\
\hline & Virola molissima & 0.57 & 0.33 & 0 & 0 & 0 & 0.03 & 0.35 \\
\hline & Virola surinamensis & 0.57 & 0.33 & 0 & 0 & 0 & 0.03 & 0.35 \\
\hline & Xylopia aromatica & 0.12 & 0 & 0 & 0 & 0 & 0 & 0 \\
\hline \multirow{22}{*}{ 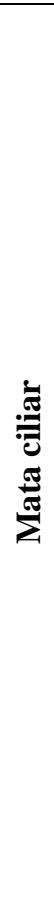 } & Andira sp. & 0.29 & 0 & 0 & 0.05 & 0.07 & 0.33 & 0.56 \\
\hline & Bixa arborea & 0 & 0 & 0 & 0 & 0 & 0 & 0.03 \\
\hline & Bunchosia sp. & 0 & 0 & 0 & 0 & 0 & 0 & 0 \\
\hline & Cassia moschata & 0.35 & 0.08 & 0 & 0.30 & 0.04 & 0.08 & 0.21 \\
\hline & Cassia sp. & 0.51 & 0 & 0 & 0 & 0.04 & 0 & 0 \\
\hline & Ceiba sp. & 0.02 & 0 & 0 & 0 & 0 & 0 & 0.09 \\
\hline & Copaifera pubiflora & 1.31 & 0.08 & 0.38 & 0.38 & 0.52 & 2.28 & 0.26 \\
\hline & Couepia multiflora & 0 & 0 & 0.11 & 0 & 0 & 0.03 & 0 \\
\hline & Curatella americana & 0.06 & 0 & 0 & 0.05 & 0.07 & 1.67 & 0.50 \\
\hline & Cynometra bauhinifolia & 0.06 & 0 & 0 & 0 & 0 & 0 & 0 \\
\hline & Erythroxylum suberosum & 0 & 0 & 0 & 0 & 0 & 0 & 0 \\
\hline & Eschweilera sp. & 1.08 & 0 & 0 & 0 & 0 & 0 & 0.06 \\
\hline & Etabalia sp. & 0.04 & 0 & 0 & 0 & 0.19 & 0 & 0 \\
\hline & Eugenia sp. & 0.33 & 0 & 0.11 & 0 & 0 & 0 & 0 \\
\hline & Genipa americana & 0.57 & 0 & 1.14 & 1.14 & 0.11 & 0.54 & 0.18 \\
\hline & Hydrochorea corymbosa & 0.25 & 0 & 0 & 0 & 0.07 & 0 & 0.12 \\
\hline & Myrcia sp. & 0.33 & 0 & 0.11 & 0 & 0 & 0 & 0 \\
\hline & Rupretchia sp. & 0 & 0 & 0 & 0 & 0 & 0 & 0 \\
\hline & Sorocea duckei & 0 & 0 & 0 & 0 & 0 & 0 & 0 \\
\hline & Spondias sp. & 0.22 & 0.08 & 0.92 & 0 & 0 & 0.23 & 0.15 \\
\hline & Swartzia sp. & 0.06 & 0 & 0 & 0.03 & 0.11 & 0 & 0 \\
\hline & Vitex schomburgkiana & 0.10 & 0 & 0.24 & 0.24 & 0.07 & 0 & 0.03 \\
\hline
\end{tabular}




\begin{tabular}{|c|c|c|c|c|c|c|c|c|}
\hline & Espécies & نُ & 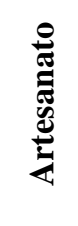 & 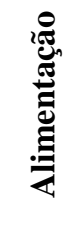 & 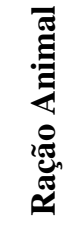 & 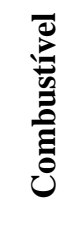 & 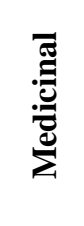 & 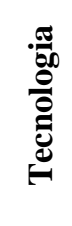 \\
\hline \multirow{11}{*}{ 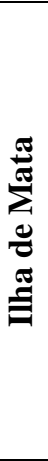 } & Anadenanthera peregrina & 0.57 & 0 & 0 & 0 & 0.04 & 0.23 & 0.24 \\
\hline & Bowdichia virgilioides & 1 & 0 & 0 & 0 & 0.33 & 0.44 & 0.15 \\
\hline & Byrsonima crassifolia & 0 & 0 & 1.46 & 0 & 1.67 & 1.18 & 0.15 \\
\hline & Copaifera pubiflora & 1.31 & 0.08 & 0.38 & 0.38 & 0.52 & 2.28 & 0.26 \\
\hline & Curatella americana & 0.06 & 0 & 0 & 0.05 & 0.07 & 1.67 & 0.50 \\
\hline & Eugenia sp. & 0.33 & 0 & 0.11 & 0 & 0 & 0 & 0 \\
\hline & Genipa americana & 0.57 & 0 & 1.14 & 1.14 & 0.11 & 0.54 & 0.18 \\
\hline & Godmania aesculifolia & 0 & 0 & 0 & 0 & 0 & 0 & 0 \\
\hline & Himatanthus draticus & 0.04 & 0 & 0 & 0 & 0.04 & 1.31 & 0.03 \\
\hline & Machaerium aculeatum & 0 & 0 & 0 & 0 & 0 & 0 & 0 \\
\hline & Xylosma benthamii & 0 & 0 & 0 & 0 & 0 & 0 & 0 \\
\hline
\end{tabular}

Tabela 6: Correlação de Pearson entre o Valor de uso e os parâmetros fitossociológicos Valor de Importância, Densidade Relativa e Dominância Relativa.

\begin{tabular}{lcccc} 
& Parâmetro Fitossociológico & r & P \\
\hline \multirow{3}{*}{ Lavrado } & & VI & 0.79 & $<0.01$ \\
& VU & DR & 0.65 & 0.05 \\
& & DoR & 0.81 & $<0.01$ \\
Buritizal & FR & 0.88 & $<0.01$ \\
& & VI & 0.62 & 0.004 \\
& & DR & 0.56 & 0.01 \\
& & DoR & 0.76 & 0.0001 \\
\hline
\end{tabular}
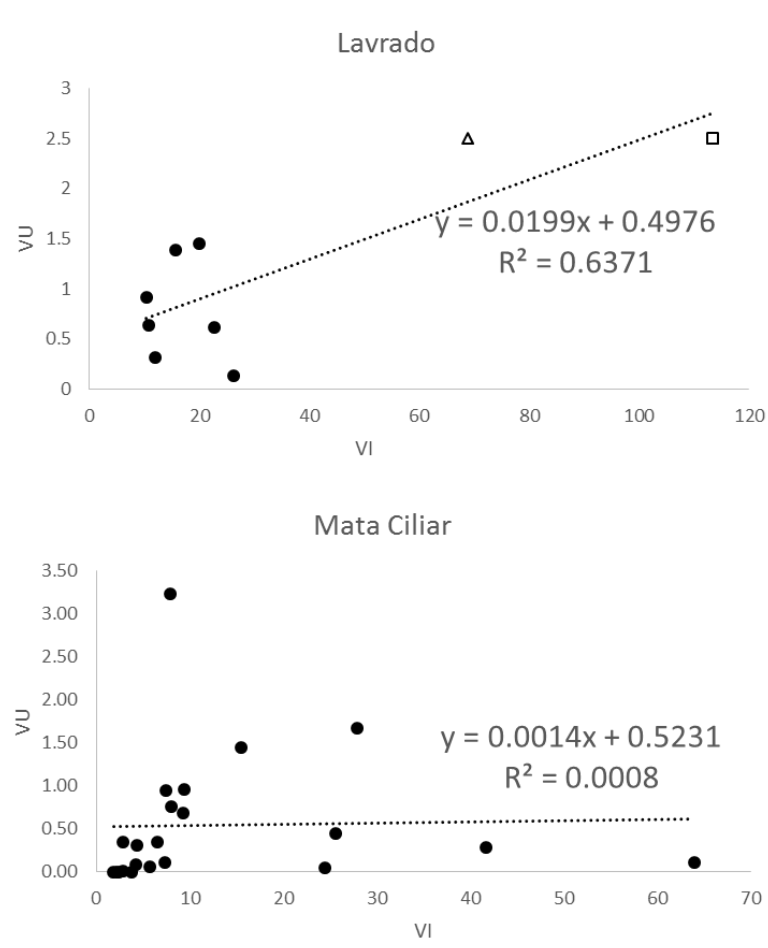

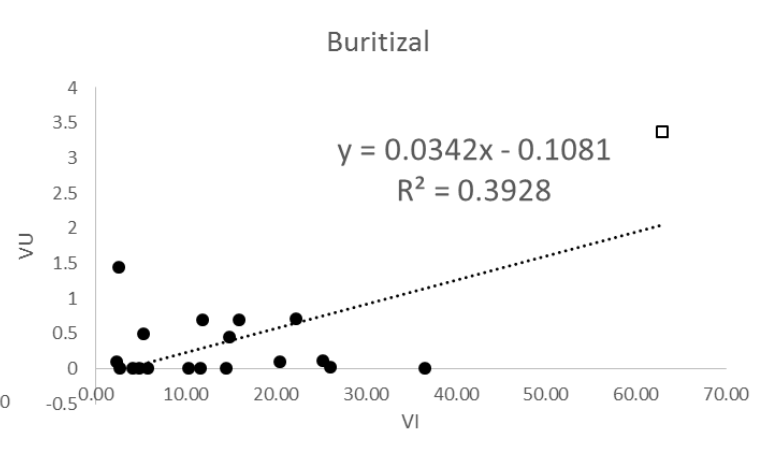

Ilha de Mata

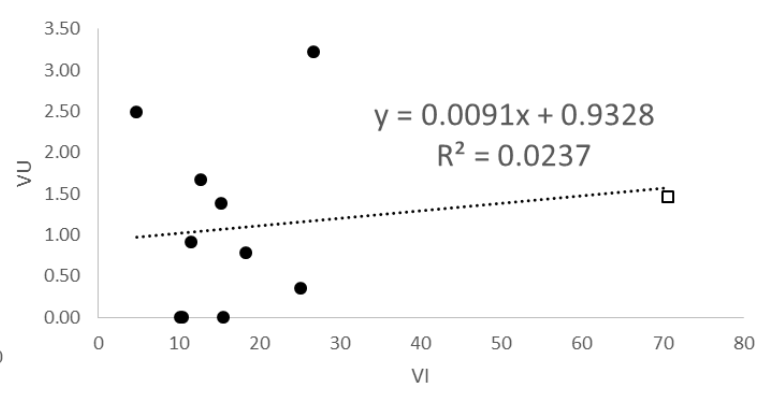

Figura 2: Correlação do VU e VI nos ambientes Lavrado, Buritizal, Mata Ciliar e Ilha de Mata na Comunidade Darora, Boa Vista, Roraima, Brasil. 
Observamos fortes associações entre o ambiente Lavrado com as categorias Alimentação e Combustível, no Buritizal com Construção, Artesanato e Alimentação com os parâmetros DR, DoR e VI, e na Ilha de Mata entre a categoria Tecnologia e DR, DoR, VI e Medicinal com DR (Tabela 7, Figura $3)$.
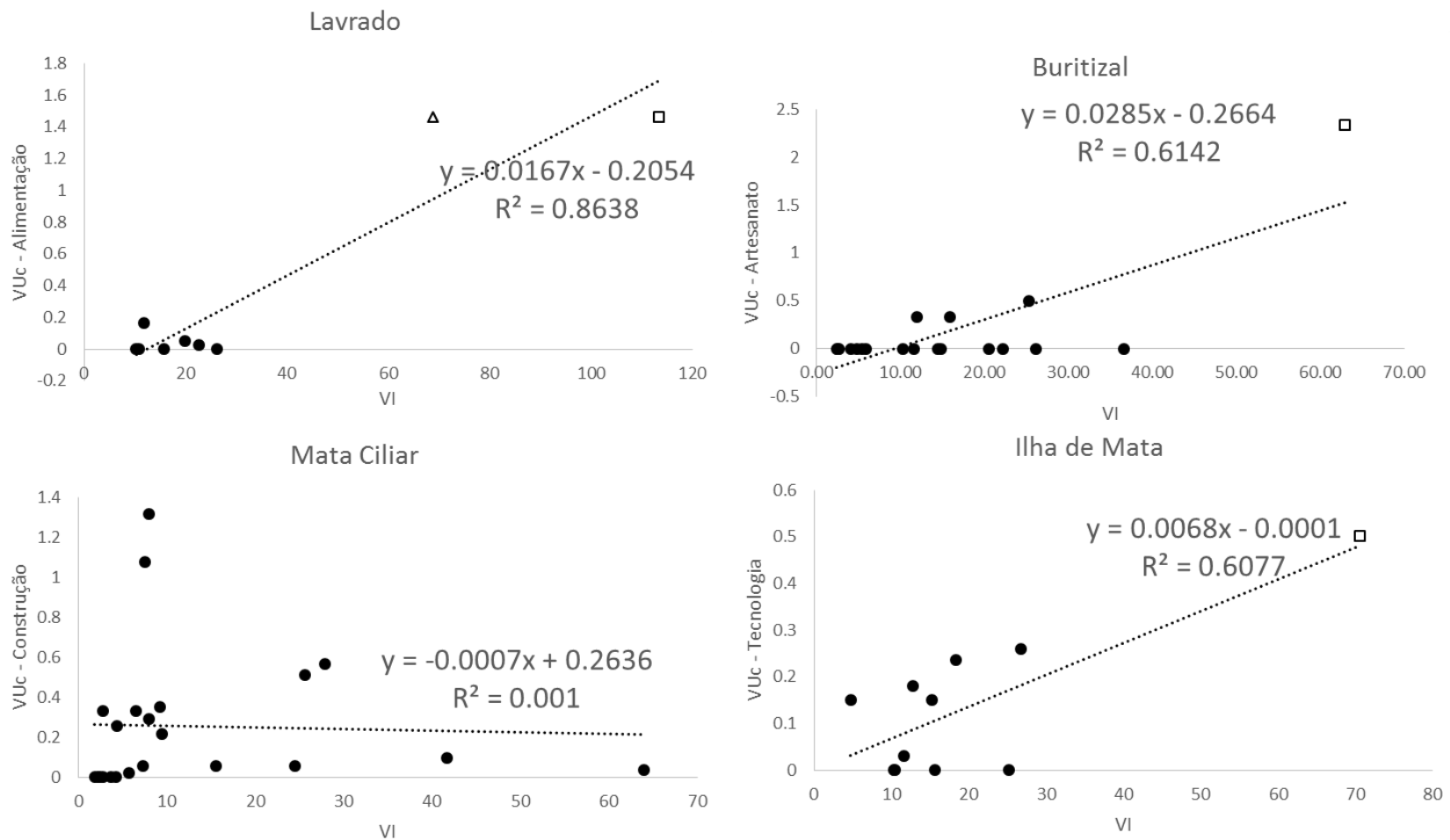

Figura 3: Correlação do VU das categorias associadas ao VI nos ambientes Lavrado, Buritizal, Mata Ciliar e Ilha de Mata na Comunidade Darora, Boa Vista, Roraima, Brasil.

As espécies de mirixi (Byrsonima crassifolia e B. coccolobifolia) destacam-se nas categorias de uso (Alimentação e Combustível) tanto pelo número de indivíduos que ambas apresentam na paisagem e consequentemente refletem na DR e DoR, quanto pela oferta de recursos, como o fruto para alimento e a lenha para serviços domésticos. Destaca-se que os mirixis também são reconhecidos como recurso medicinal pela comunidade, sendo utilizados no tratamento de diferentes distúrbios.

Da mesma forma, o buriti (M. flexuosa), no Buritizal, é bastante citado para os mais diversos usos, não sendo apenas de uso medicinal pelos participantes (Tabela 7, Figura 3). Adicionalmente, estes dados nos fazem perceber a importância do ambiente Buritizal (Maracajá) como fonte de recursos madeireiros para construção. Além do buriti, a ucuuba (Virola molissima e V. surinamensis), o miri (Humiria balsamifera) e o açaí (Euterpe precatoria) também são reconhecidos como espécies utilizadas na construção, especificamente como madeira para caibros, ripas e travessas. E ainda, há a forte relação do buriti como recurso para artesanato, na confecção de trajes típicos para danças e na confecção de brinquedos a partir do caranã (pecíolo), bem como na alimentação com o consumo do buriti e também do açaí, principalmente.

$\mathrm{Na}$ Ilha de Mata, a forte associação da categoria Tecnologia e Medicinal com os parâmetros fitossociológicos está relacionada ao caimbé (Curatella americana), principalmente utilizado na confecção de bancos e utensílios para caça e pesca, e para o tratamento de doenças, e também a copaíba (Copaifera pubiflora), cujo uso principal é a produção de tábuas para móveis e por ser uma das espécies medicinais principais da comunidade (Figura 3, Tabela 7). 
Tabela 7: Correlação de Pearson entre as categorias de uso nos ambientes e os parâmetros fitossociológicos, Comunidade Darora, Boa Vista, Roraima.

\begin{tabular}{|c|c|c|c|c|}
\hline & Categoria de uso & $\begin{array}{c}\text { Parâmetro } \\
\text { Fitossociológico }\end{array}$ & $\mathbf{r}$ & $\mathbf{P}$ \\
\hline \multirow{8}{*}{ Lavrado } & \multirow{4}{*}{ Alimentação } & DR & 0.86 & 0.002 \\
\hline & & DoR & 0.88 & 0.001 \\
\hline & & FR & 0.98 & $5.98 \mathrm{E}-07$ \\
\hline & & VI & 0.92 & 0.0002 \\
\hline & \multirow{4}{*}{ Combustível } & DR & 0.86 & 0.002 \\
\hline & & DoR & 0.88 & 0.001 \\
\hline & & FR & 0.98 & $9.88 \mathrm{E}-07$ \\
\hline & & VI & 0.92 & 0.0003 \\
\hline \multirow{9}{*}{ Buritizal } & \multirow{3}{*}{ Construção } & DR & 0.64 & 0.002 \\
\hline & & DoR & 0.79 & $4.0376 \mathrm{E}-05$ \\
\hline & & VI & 0.70 & 0.0007 \\
\hline & \multirow{3}{*}{ Artesanato } & DR & 0.69 & 0.0009 \\
\hline & & DoR & 0.89 & 2.0404E-07 \\
\hline & & VI & 0.78 & 7.1951E-05 \\
\hline & \multirow{3}{*}{ Alimentação } & DR & 0.69 & 0.0009 \\
\hline & & DoR & 0.85 & $3.3317 \mathrm{E}-06$ \\
\hline & & VI & 0.73 & 0.0003 \\
\hline \multirow{4}{*}{$\begin{array}{l}\text { Ilha de } \\
\text { Mata }\end{array}$} & \multirow{3}{*}{ Tecnologia } & DR & 0.78 & 0.004 \\
\hline & & DoR & 0.79 & 0.003 \\
\hline & & VI & 0.83 & 0.001 \\
\hline & Medicinal & DoR & 0.62 & 0.03 \\
\hline
\end{tabular}

\section{DISCUSSÃO}

Sessenta e nove por cento das espécies amostradas no levantamento fitossociológico foram reconhecidas pelos moradores ( 36 das 52 espécies), valor inferior aos 95\% registrados por Alarcón e Peixoto (2008) em estudo realizado na Comunidade Caicubi, no sul do Estado de Roraima, porém, podemos considerá-lo mais próximo aos estudos realizados com diferentes etnias indígenas na Amazônia brasileira, como os Kayapó (76,8\%), os Tembé (61,3\%) e os Waimiri-Atroari (79\%), além dos Panaré (48,6\%) na Venezuela e os Chácobo (78,7\%) na Bolívia (PRANCE et al., 1987; MILLIKEN et al., 1992).

Em estudo realizado no Cerrado brasileiro, Tunholi et al. (2013) registraram que a população local reconheceu 49,3\% das espécies inventariadas, e Lucena et al. (2007) registraram que 92\% das espécies inventariadas eram consideradas úteis por duas comunidades rurais na Caatinga. Apesar da Comunidade Darora demonstrar uma forte relação com as plantas de seu meio, este percentual de espécies inventariadas/úteis pode variar de acordo com as características da vegetação e com a relação dos moradores com o seu respectivo ambiente (LADIO et al., 2007; THOMAS, 2012).

\section{Aparência ecológica}

Os moradores da comunidade Darora citaram poucos usos para a maioria das espécies vegetais e poucas espécies foram muito citadas. Este é um padrão também encontrado em outros estudos (GALEANO, 2000; FERRAZ et al., 2006; LUCENA et al., 2007; LIMA et al., 2012; TUNHOLI et al., 2013). Esse padrão parece referir a ambientes com alta biodiversidade, pois uma vez que a riqueza de espécies aumenta, pode ocorrer o aparecimento de espécies raras, pouco conhecidas e com menos usos pelas populações locais (SHEIL e SALIM, 2012). Da mesma forma, podemos também observar que nos ambientes Lavrado e Buritizal, as espécies de mirixi (Byrsonima crassifolia e B. coccolobifolia) e buriti (Mauritia flexuosa), respectivamente, apresentam os maiores valores de uso, bem como os maiores valores fitossociológicos, além de uma forte associação entre essas variáveis. Deste modo, o presente estudo corrobora a hipótese da Aparência Ecológica, onde as pessoas usam as espécies mais aparentes de seu ambiente, e se equivale a outros estudos (TORRE-CUADROS e ISLEBE, 2003; LUCENA et al., 2007; GUÈZE et al., 2014). 
Por outro lado, a relação VI e VU não foi positiva no ambiente Mata Ciliar (área amostral de 0,5 ha), embora que mututi (Etabalia sp.) e maria-preta (Vitex schomburgkiana), mesmo apresentando um dos maiores VI da amostra, não apresentaram VU suficientes para corroborar a hipótese. Vale ressaltar que valores ecológicos podem ser tendenciosos devido ao tamanho das amostras e ao diâmetro dos indivíduos (MACIA, 2008), uma vez que ambas as espécies apresentaram os valores de DoR próximos de $50 \%$ de seus respectivos VI. A Mata Ciliar também apresentou espécies endêmicas com apenas um indivíduo e VU muito baixo (Bixa arborea, Couepia multiflora, Hydrochorea corymbosa e Myrcia sp.), além de espécies com dois ou três indivíduos. Tais espécies, apesar de não apresentarem $\mathrm{VI} \leq 1$, apresentaram poucos ou apenas um indivíduo (estas últimas consideradas "espécies raras"), o que pode ter ocasionado uma relação negativa de VU/VI (GUĖZE et al., 2014). Vale ressaltar que a limitação do VI, onde o alto valor de uma das variáveis (DR, DoR e FR) em relação às outras, pode mascarar o significado real de determinada espécie no ambiente.

A Ilha de Mata com área amostral de 0,25 ha apresentou apenas oito espécies e 42 indivíduos úteis. É um tipo de ambiente da savana de Roraima que apresenta um histórico de perturbação pelas populações locais, onde são empregadas atividades agrícolas, extração de madeira e pisoteio do subbosque pelo gado, e ainda pela ação do fogo que pode influenciar na riqueza e diversidade de espécies (LAWRANCE et al., 2000; SANTOS et al., 2013). Observamos que, por apresentar valores numéricos distintos entre VU e VI, como o caimbé (Curatella americana) que apresentou um alto VI e baixo VU, bem como mirixi e copaíba por apresentarem um alto VU e baixo VI, não podemos confirmar a hipótese da Aparência neste ambiente. É possível que, no passado, este foi também um ambiente de uso pelos moradores, mas que pode ter sido esquecido por se localizar distante do centro da vila e ter em sua oferta de recursos espécies que estão presentes em ambientes mais próximos aos moradores.

Este caso nos remete a outro ponto da hipótese da aparência ecológica, que seria a causalidade reversa, onde manejo e utilização deste ambiente podem ter influenciado a sua importância no passado (THOMAS et al., 2009), e a composição florística e a abundância atuais podem ser resultantes das modificações ocorridas (MACIA 2008; GUÈZE et al., 2014), pois espécies como copaíba (Copaifera pubiflora) e angico (Anadenanthera peregrina) parecem ter sido fortemente exploradas e podem ter tido suas disponibilidades diminuídas na comunidade ao longo dos anos. Neste sentido, podemos destacar a espécie arbórea que nomeia a comunidade, a darora (Leptolobium nitens Vogel), que sofreu uma forte pressão extrativista no passado, não foi registrada em nosso levantamento fitossociológico, mas é uma espécie citada nas entrevistas pelo seu uso madeireiro e quase sempre indicada primeiramente pelos moradores por ser o nome da Comunidade.

\section{Valor de uso das categorias}

No Lavrado, encontramos uma forte correlação entre as categorias Alimentação e Combustível com os quatro parâmetros fitossociológicos. A categoria Alimentação, bem como a categoria Medicinal, normalmente não confirmam a hipótese da Aparência Ecológica. No caso, esta foi confirmada apenas no estudo realizado por Philips e Gentry (1993). A categoria Combustível está bastante relacionada à DoR, como encontrado por Lucena et al. (2007) e Ribeiro et al. (2014) na Caatinga, bem como registradas por Thomas et al. (2009) na Bolívia, onde $60 \%$ das espécies são usadas como combustível.

O buriti (M. flexuosa) apresenta-se como uma espécie de múltiplos usos e está presente no cotidiano dos moradores. As palmeiras estão entre as espécies de mais fácil identificação na Amazônia e na vegetação savânica como registrado em outros estudos (BYG e BALSLEV, 2001; MARTINS et al., 2014). São elementos característicos da paisagem dos lavrados em Roraima, indicando algum curso de rio ou igarapé. Assim como outras palmeiras, são facilmente vistos, identificados e utilizados.

Na Ilha de Mata, observamos uma relação positiva de Tecnologia com os parâmetros DoR e VI, e Medicinal com a DoR, evidenciando a seleção das espécies por conta da ocupação diamétrica/área basal dos indivíduos neste ambiente, concordando com Lawrence et al. (2005), que sugerem que a aparência ecológica está mais ligada à dominância das espécies. Localmente, as atividades madeireiras para a retirada de tábuas para móveis e a utilização das cascas do caule para o preparo de medicamentos são bastante comuns na comunidade.

Foi encontrada uma associação negativa no ambiente Mata Ciliar, que pode advir do fato do baixo número de indivíduos das espécies, bem como o baixo VU. Esta situação pode evidenciar a preferência de uso por outras espécies localizadas no Buritizal, como exemplo, uma vez que este ambiente é mais distante e suas espécies obtiveram uma correlação positiva, mas não significativa nas categorias Alimentação e Combustível.

\section{CONCLUSÕES}


Os indígenas da Comunidade Darora possuem grande conhecimento sobre as espécies lenhosas e suas indicações de uso e, desta forma, a Aparência Ecológica foi corroborada nos ambientes Lavrado e Buritizal, bem como houve uma correlação positiva entre os parâmetros fitossociológicos com o VU das categorias Alimentação e Combustível no ambiente Lavrado, das categorias Alimentação, Artesanato e Construção no ambiente Buritizal, e das categorias Medicinal e Tecnologia no ambiente Ilha de Mata.

O estudo também apresentou poucas espécies com muitos usos e muitas espécies com poucos usos, padrão muito comum em estudos etnobotânicos. E espécies com muitos usos, mas com baixo valor ecológico, o que de certo modo pode ocasionar em sobre-exploração das espécies mais preferidas, como o caso da espécie darora, que dá nome à comunidade, mas não foi encontrada na amostragem realizada. Desta forma, o ambiente Buritizal também é merecedor de uma maior atenção, uma vez que suas espécies são muito usadas para fins madeireiros.

O estudo da relação disponibilidade local x valor de uso se constitui numa importante ferramenta capaz de fornecer informações reais e necessárias das comunidades, como potencial de uso das espécies, possíveis espécies e ambientes que podem estar sofrendo algum risco nos ambientes, subsidiar estratégias de manejo e conservação dos recursos.

\section{REFERÊNCIAS}

AGUIAR JR, A.; BARBOSA, R.I.; BARBOSA, J.B.F.; MOURÃO J.R.M. Invasion of Acacia mangium in Amazonian savannas following planting for forestry. Plant Ecology and Diversity, 7: 359-369, 2014.

ALARCÓN, J.G.S.; PEIXOTO, A.L. Use of terra firme Forest by Caicubi Caboclos, Middle Rio Negro, Amazonas, Brazil. A quantitative study. Economic Botany, 62(1): 60-73, 2008.

ALBUQUERQUE, U.P. Etnobotânica aplicada à conservação da biodiversidade. In: ALBUQUERQUE U.P.; LUCENA, R.F.P.; CUNHA L.V.F.C. (orgs). Métodos e técnicas na pesquisa etnobiológica e etnoecológica. Recife, Nupeea. p. 351-363, 2010.

ALBUQUERQUE, U.P.; LUCENA, R.F.P. Can apparency affect the use of plants by local people in tropical forests. Interciencia 30(8): 506-511, 2005.

ALBUQUERQUE, U.P.; LUCENA, R.F.P.; LINS-NETO, E.M.F. Selection of research participants. In: ALBUQUERQUE, U.P.; CUNHA, L.V.F.C.; LUCENA, R.F.P.; ALVES, R.R.N. (ed). Methods and techniques in Ethnobiology and Ethnoecology New York: Springer Protocols Handbooks, p. 1-13, 2014.

ANGIOSPERM PHYLOGENY GROUP IV. An update of the Angiosperm Phylogeny Group classification for orders and families of flowering plants: APG IV. Botanical Journal of the Linnean Society, 181: 1-20, 2016.

AYANTUDE, A.A.; HIERNAUX, P.; BRIEJER, M.; UDO, H.; TABO, R. 2009. Uses of local plant species by agropastoralists in south-western Niger. Etnobotany Research e Applications, 7: 53-66.

BRANDT, R.; MATHEZ-STIEFEL, S.; LACHMUTH, S.; HENSEN, I.; RIST, S. 2013. Knowledge and valuation of Andean agroforestry species: the role of sex, age, and migration among members of a rural community in Bolivia. Jounal of Ethnobiology and Ethnomedicine, 9(1): 83.

BARBOSA, R.I.; CAMPOS, C.; PINTO, F.; FEARNSIDE, P.M. 2007. The "lavrados" of Roraima: Biodiversity and Conservation of Brazil's Amazonian Savannas. Functional Ecosystems and Communities 1(1): 29-41.

BARBOSA, R.I.; NASCIMENTO, S.P.; AMORIM, P.A.F.; SILVA, R.F. Notas sobre a composição arbóreoarbustiva de uma fisionomia das savanas de Roraima, Amazônia Brasileira. Acta Botanica Brasilica, 19: 323329, 2005.

BARBOSA, R.I. Distribuição das chuvas em Roraima. In: BARBOSA, R.I., FERREIRA, E.J., CASTELLON, E.G. (eds.). Homem, Ambiente e Ecologia no Estado de Roraima. Manaus Instituto Nacional de Pesquisas da Amazônia, p. 325- 335, 1997.

BYG, A.; BALSLEV, H. 2001. Diversity and use of palms in Zahamena, eastern Madagascar. Biodiversity and Conservation, 10: 951-970, 2001.

CORDEIRO, A.C. O cultivo arroz irrigado em Roraima In: BARBOSA, R.I., XAUD, H.A.M., SOUZA, J.M.C. Savanas de Roraima: etnoecologia, biodiversidade e potencialidades agrossilvipastoris. Boa Vista: FEMACT, p.169-176, 2005.

CUNHA, L.V.F.C.; ALBUQUERQUE, U.P. Quantitative ethnobotany in an Atlantic Forest fragment of Northeastern Brazil - Implications to conservation. Environmental Monitoring and Assessment, 114: 1-25, 2006.

FERRAZ, J.S.F.; ALBUQUERQUE, U.P.; MEUNIER, I.M.J. Use-value and phytosociology of woody plants on the banks of the Riacho do Navio stream, Floresta, Pernambuco State, Brazil. Acta Botanica Brasilica, 20: 125-134, 2006.

FLORES A.S.; RODRIGUES R.S. Diversidade de Leguminosae em uma área de savana do estado de Roraima, Brasil. Acta Botanica Brasilica, 24: 175-183, 2010. 
GALEANO, G. Forest use at the Pacific Coast of Chocó, Colômbia: a quantitative approach. Economic Botany, 54: 358-376, 2000.

GIANLUPPI, D.; SMIRDELE, O.J. O cultivo da soja nos cerrados de Roraima. In: BARBOSA, R.I., XAUD, H.A.M., SOUZA, J.M.C. Savanas de Roraima: etnoecologia, biodiversidade e potencialidades agrossilvipastoris. Boa Vista: FEMACT, 2005. p.177-182, 2005.

GUÈZE, M.; LUZ, A.C.; PANEQUE-GÁLVEZ, J.; MACÍA, M.J.; ORTA-MARTÍNEZ, M.; PINO, J.; REYSGARCÍA, V. Are ecologically important tree species the most useful? A case study from indigenous people in the Bolivian Amazon. Economic Botany, 20: 1-15, 2014.

JIMÉNEZ-ESCOBAR, N.D.; RANGEL, J.O. La abundancia, la dominancia y sus relaciones con el uso de la vegetación arbórea en la Bahía de Cispatá, Caribe Colombiano. Caldasia, 34: 347-366, 2012.

LADIO, A.; LOZADA, M.; WEIGANDT, M. Comparison of traditional wild plant knowledge between aboriginal communities inhabiting arid and forest environments in Patagonia, Argentina. Journal of Arid Enviroments, 69: 695-715, 2007.

LAWRANCE, W.F.; VASCONCELOS, H.L.; LOVEJOY, T.E. Forest loss and fragmentation in the Amazon: implications for wildlife conservation. Oryx 34: 39-45, 2000.

LAWRENCE, A.; PHILLIPS, O.L.; ISMODES, A.R.; LOPEZ, M.; ROSE, S.; WOOD, D.; FARFAN, A.J. Local values for harvested forest plants in Madre de Dios, Peru: towards a more contextualised interpretation of quantitative ethnobotanical data. Biodiversity and Conservation, 14: 45-79, 2005.

LIMA, I.L.P.; SCARIOT, A.; MEDEIROS, M.B.; SEVILHA, A.C. Diversidade e uso de plantas do Cerrado em comunidades de Geraizeiros no norte do estado de Minas Gerais, Brasil. Acta Botanica Brasilica, 26: 675684, 2012.

LUCENA, R.F.P.; ARAÚJO, E.L.; ALBUQUERQUE, U.P. 2007. Does the local availability of woody Caatinga plants (Northeastern Brazil) explain their use value? Economic Botany, 61: 347-361, 2007.

LUCENA, R.F.P.; MEDEIROS, P.M.; ARAÚJO, E.L.; ALVES, A.G.C.; ALBUQUERQUE, U.P. The ecological apparency hypothesis and the importance of useful plants in rural communities from Northeastern Brazil: an assessment based on use value. Journal of Enviromental Management, 96:106-115, 2012.

MACÍA M.J. Woody plants diversity, floristic composition and land use history in the Amazonian rain forests of Madidi National Park, Bolivia. Biodiversity and Conservation, 17:2671-2690, 2008.

MALDONADO, B.; CABALLERO, J.; DELGADO-SALINAS, A.; LIRA, R. Relationship between use value and ecological importance of floristic resources of seasonally dry tropical forest in the Balsas river basin, México. Economic Botany, 67: 17-29, 2013.

MARTINS, R.C.; FILGUEIRAS, T.S.; ALBUQUERQUE, U.P. Use and diversity of palm (Arecaceae) resources in Central Western Brazil. The Scientific World Journal. Article ID 942043, 14 p, 2014.

MELO, M.C.; BARBOSA, R.I. Árvores e arbustos das savanas de Roraima: guia de Lavrado ilustrado. 1. ed. Boa Vista: PMBV/CONSEMMA, 2007.

MILLIKEN, W.; MILLER, R.P.; POLLARD, S.R.; WANDELLI, E.V. Ethnobotany of the Waimiri Atroari Indians of Brazil. London, Kew: Royal Botanic Gardens. 146p, 1992.

OLIVEIRA, R.L.C. Uso e conhecimento das espécies lenhosas em uma Comunidade Indígena na savana de Roraima. 2016 (Tese de doutorado - Botânica) - Programa de Pós-graduação em Botânica. Instituto Nacional de Pesquisas da Amazônia, 2016.

PHILLIPS, O.; GENTRY, A.H. The useful plants of Tambopata, Peru: I statistical hypothesis test with a new quantitative. Economic Botany 47, p. 15-32, 1993 a.

PHILLIPS, O.; GENTRY, A.H. The useful plants of Tambopata, Peru: II. Additional hypothesis testing in quantitative ethnobotany. Economic Botany, 47: 33-43, 1993 b.

PRANCE, G.T.; BALÉE, W.; BOOM, B.M.; CARNEIRO, R.L. Quantitative ethnobotany and the case for conservation in Amazonia. Conservation Biology 1: 296-310, 1987.

REYES-GARCÍA, V.; HUANCA, T.; VADEZ, V.; LEONARD, W.; WILKIE, D. Cultural, practical, and economic value of wild plants: a quantitative study in the Bolivian Amazon. Economic Botany, 60 (1): 62-74, 2006.

RIBEIRO, J.P.O.; CARVALHO, T.K.N.; RIBEIRO, J.E.S.; LIMA, J.R.F.; SOUSA, R.F.; ALVES, C.B.; JARDIM, J.G.; LUCENA, R.F.P. Can ecological apparency explain the use of plant Species in the semi-arid depression of Northeastern Brazil? Acta Botanica Brasilica, 28: 476-483, 2014.

RIBEIRO, J.E.L.S.; HOPKINS, M.J.G.; VICENTINI A.; SOTHERS, C.A.; COSTA, M.A.S.; BRITO, J.M.; SOUZA, M.A.D.; MARTINS, L.H.P.; LOHMANN, L.G.; ASSUNÇÃO, P.A.C.L.; PEREIRA, E.C.; SILVA, C.F.; MESQUITA, M.R.; PROCÓPIO, L.C. Flora da Reserva Ducke: guia de identificação das plantas vasculares de uma floresta de terra-firme na Amazônia Central. Manaus, INPA/DFID. 1999.

ROSSATO, S.C.; LEITÃO FILHO, H.; BEGOSSI, A. Ethnobotany of Caiçaras of the Atlantic Forest coast (Brazil). Economic Botany, 53(4): 387-395, 1999.

SANTOS, N. M. C.; VALE-JUNIOR, J. F.; BARBOSA, R. I. Florística e estrutura arbórea de ilhas de mata em áreas de savana no norte da Amazônia brasileira. Bol. Mus. Paraense Emílio Goeldi Ciências Naturais 8(2): 205-221, 2013. 
SHEIL, D.; SALIM, A. Diversity of locally useful tropical Forest wild-plants as a function of species richness and informant culture. Biodiversity and Conservation, 21: 687-699, 2012.

THOMAS, E.; VANDEBROEK, I.; VAN DAMME, P.; GOETGHEBEUR, P.; DOUTERLUNGNE, D.; SANCA, S.; ARRAZOLA, S. The relation between accessibility, diversity and indigenous valuation of vegetation in the Bolivian Andes. Journal of Arid Environments, 73: 854-861, 2009.

THOMAS, E. The impact of traditional lifestyle, provenance and contact history on plant use knowledge and management: a cross-cultural comparison of two small-scale societies from the Bolivian Amazon. Human Ecology, 40: 355-368, 2012.

TORRES-CUADROS, M.L.A.; ISLEBE, G.A. Traditional ecological knowledge and use of vegetation in southeastern Mexico: a case study from Solferino, Quintana Roo. Biodiversity and Conservation, 12: 2455-2476, 2003

TUNHOLI, V.P.; RAMOS, M.A.; SCARIOT, A. Availability and use of woody plants in an agrarian reform settlement in the Cerrado of the state of Goiás, Brazil. Acta Botanica Brasilica, 27(3): 604-612, 2013.

WITTMANN, F.; SCHONGART, J.; DE BRITO, J.M.; WITTMANN, A.O.; PIEDADE, M.T.F.; PAROLIN, P.; JUNK, W.J.; GUILlAUMET, J.L. Manual of trees from Central Amazonian varzea floodplains: taxonomy, ecology and use. 1. ed. Manaus, Editora INPA, 2010. 